\title{
The usage of optical coupler and its optimization signal in modified optical time domain reflectometer
}

\author{
Subramaniam Jahanadan* and Yusof Munajat \\ Advanced Photonics Science Institute, Department of Physics, Faculty of Science, Universiti Teknologi Malaysia, \\ 81310 UTM Skudai, Johor, Malaysia \\ Received 25 March 2011, Revised 5 April 2011, Accepted 10 May 2011, Available online 20 May 2011.
}

\begin{abstract}
In this study, attention was made to measure the length of the fiber and the coefficient of the fiber attenuation utilizing novel configurations. Laser diode source with a fundamental wavelength of $(808 \pm 10) \mathrm{nm}$ with a maximum power of $50 \mathrm{~mW}$ was employed. First measurement utilizes a polarized beam splitter whereas the second measurement utilizes a beam coupler. The laser was launched in pulsed mode with a 75 ns pulse width which delivered approximately $15 \mathrm{~mW}$ power into the fiber and the laser source was adjusted to operate in continuous mode with a $50 \%$ duty cycle. A preamplified Si PIN photodetector was used to detect the backscattered light. Later these results were compared with a measurement that utilizes a conventional Anritsu OTDR with a $850 \mathrm{~nm}$ wavelength. The length of the fiber obtained is $1313.99 \mathrm{~m}$ and shows a deviation of $0.46 \%$ for the first measurement and 1303.89 $\mathrm{m}$ with a deviation of $0.38 \%$ for the second measurement. The attenuation coefficient for that fiber is $2.65 \mathrm{~dB}^{\mathrm{k}} \mathrm{km}^{-1}$ with a deviation of $16.22 \%$ for the first measurement and $2.58 \mathrm{~dB} \cdot \mathrm{km}^{-1}$ with a deviation of $13.16 \%$ for the second measurement. An adjustable BK7 glass plate was used for signal optimization in the system. Results show that maximum coupling and also maximum backscattered signal occurs when the BK7 glass window is rotated $35^{\circ}$ anticlockwise.
\end{abstract}

| Fiber Length | Attenuation | OTDR | Signal Optimization |

(B) $2011 \mathrm{Ibnu}$ Sina Institute. All rights reserved. http://dx.doi.org/10.11113/mjfas.v7n1.206

\section{INTRODUCTION}

The evolution of Optical Time Domain Reflectometer (OTDR) followed from a publication by Barnoski and Jensen that described backscattering phenomenon in optical fibers and illustrated its potential use for fiber characterization back in 1976 [1,2]. The technique of OTDR has a primary advantage of not requiring any cuts in the fiber as it works by measuring backscattered light rather than transmitted light $[2,3]$. This backscattered information can be used to measure loss in cable plants as well as to locate fiber breaks, splices and connectors. Rayleigh scattering and Fresnel reflection principally produce the backscattered light which shall be discussed in brief [2].

The objective of this research is to develop an OTDR system to determine the length and attenuation of a multimode optical fiber by using a fiber coupler and a beam splitter. Later this result was compared with a conventional Anritsu OTDR. The influence of the BK7 glass window on coupling efficiency and backscattered signal was also investigated.

Corresponding author at: Advanced Photonics Science Institute, Department of Physics, Faculty of Science, Universiti Teknologi Malaysia, 81310 UTM Skudai, Johor, Malaysia

E-mail addresses: jsubra82@gmail.com

\section{THEORY}

Fresnel reflection concerns about the amount of light reflection which occurs at a plane boundary between two dielectrics. The reflection coefficient, $r$ is the ratio of the reflected electric field to the incident electric field. For normal incidence, the Fresnel's equation simplifies into;

$$
r=\frac{n_{1}-n_{2}}{n_{1}+n_{2}}
$$

where $n_{1}$ is the refractive index in the incident region and $n_{2}$ is the index in the transmitted region. If $n_{2}>n_{1}$, the reflection coefficient becomes negative and this indicates a $180^{\circ}$ phase shift between the incident and the reflected electric fields $[4,5]$. The reflectance, $R$ is the ratio of the reflected-beam intensity to the incident-beam intensity. Because the intensity in an optic beam is proportional to the square of its electric field, the reflectance is equal to the square of the reflection coefficient. Thus,

$$
R=\left(\frac{n_{1}-n_{2}}{n_{1}+n_{2}}\right)^{2}
$$


A perfect fiber end reflects about 4 percent of the power incident on it.

Rayleigh scattering reflects light in all directions throughout the length of the fiber. This factor is the dominant loss mechanism in most high-quality fibers. Suppose the input optical power into a fiber of length, $L$ is $P_{\text {in }}$ and the output optical power at the destination end is $P_{\text {out }}$ and intensity anywhere in the fiber at a distance $x$ from the input is $P$. The attenuation coefficient, $\alpha_{d B}$ is defined as the fractional decrease in the optical power per unit distance;

$$
\alpha_{d B}=-\frac{1}{P} \frac{d P}{d x}
$$

We can integrate this over the length, $L$ of the fiber to relate $\alpha$ to $P_{\text {out }}$ and $P_{\text {in }}$ by;

$$
\alpha_{d B}=-\frac{1}{L} \ln \left(\frac{P_{\text {out }}}{P_{\text {in }}}\right)
$$

We can just as well define attenuation in terms of light intensity but we have used power to follow convention since attenuation tests on optical fibers measure the optical power. In general, optical power attenuation in an optical fiber is expressed in terms of decibels per unit length of fiber, typically dB per km [5]. The attenuation of the signal in decibels per unit length is defined in terms of the $\underline{\text { logarithm to base } 10}$ by;

$\alpha_{d B}=-\frac{1}{L} 10 \log \left(\frac{P_{\text {out }}}{P_{\text {in }}}\right)$

In addition, to determination of attenuation and component losses, an OTDR also can be used to measure length and to locate breaks in an optical fiber. The fiber length, $L$ can be calculated from the time difference between the pulses reflected from the front and far ends of the fiber. If this time difference is $t$, then the length $L$ is given by;

$$
L=\frac{c t}{2 n}
$$

where $n$ is the refractive index of the fiber core. The factor 2 accounts for the round trip made by the pulse [4].

\section{EXPERIMENTAL METHOD}

The set-up for the polarized beam splitter is shown in Figure 1, whereas the modification set-up with a beam coupler is set up as shown in Figure 2 without an adjustable BK7 glass window [6]. For length measurement, the controller unit is set to operate the diode laser in the pulsed mode. The laser pulse width is $75 \mathrm{~ns}$ with energy of $1 \mathrm{~nJ}$ per pulse and the average power of the laser is $15 \mathrm{~mW}$.

In order to measure the value of attenuation coefficient, the control unit is set to $50 \%$ duty cycle to operate the laser diode in continuous mode. The number of photons launched into the optical fiber was increased by increasing the current on the laser diode controller or by enlarging the pulse length. During the phase of the switched on radiation, one fills the fibers with photons which produce the scattering light at the Rayleigh scattering centers which is directed towards the fiber entrance. The switching off the light pulse results in a rapid reduction of the backscattering light produced at the fiber entrance surface.

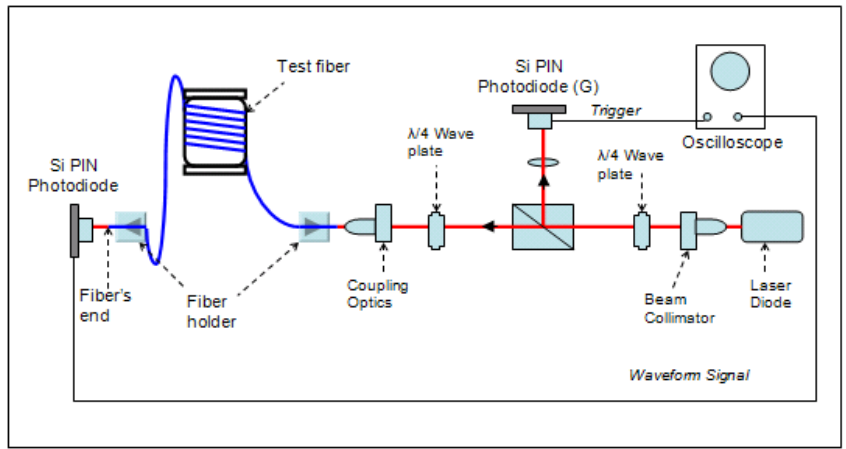

Fig. 1. Schematic Diagram of Experimental Set Up with a polarized beam splitter

The setup for the signal optimization is shown in Figure 2. The BK7 glass window was rotated anticlockwise by a variance of $5^{\circ}$ from $0^{0}$ to $55^{\circ}$. The coupling voltage difference, $\Delta V$ at Port 2 and Port 3 which fixed on Oscilloscope was measured and recorded. The return signal voltage difference, $\Delta V$ at Port 4 was measured and recorded for further analysis.

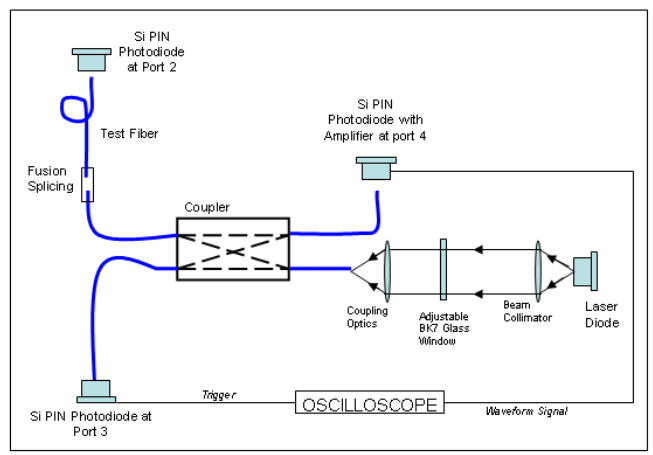

Fig. 2. Schematic Diagram of Experimental Set Up of OTDR with adjustable BK7 glass Window

\section{RESULTS AND DISCUSSION}

The fiber length measurement using a polarized beam splitter is $(1314 \pm 21) \mathrm{m}$, whereas with the beam coupler the fiber length is $(1304 \pm 11) \mathrm{m}$.

The typical result obtained for the measurement of the optical fiber attenuation using a continuous wave with a 
polarized beam splitter is shown in Figure 3. The gradient of the graph contains the loss or attenuation coefficient. Calculating the gradient for selected point $\mathrm{A}$ and point $\mathrm{B}$ on the graph yields attenuation coefficient, $\alpha_{d B}=(2.65 \pm$ $0.03) \mathrm{dB} \cdot \mathrm{km}^{-1}$. Analyzing graph in Figure 3 shows that there is a sudden loss at the end of fiber. The studied fiber was packaged in a reel of drum and this could have caused the light ray to escape from fiber due to the difference in radius of the fiber reel [7]. Besides macrobend, crack in the fiber or manufacturing default also could be a possible contributor for the abrupt signal loss.

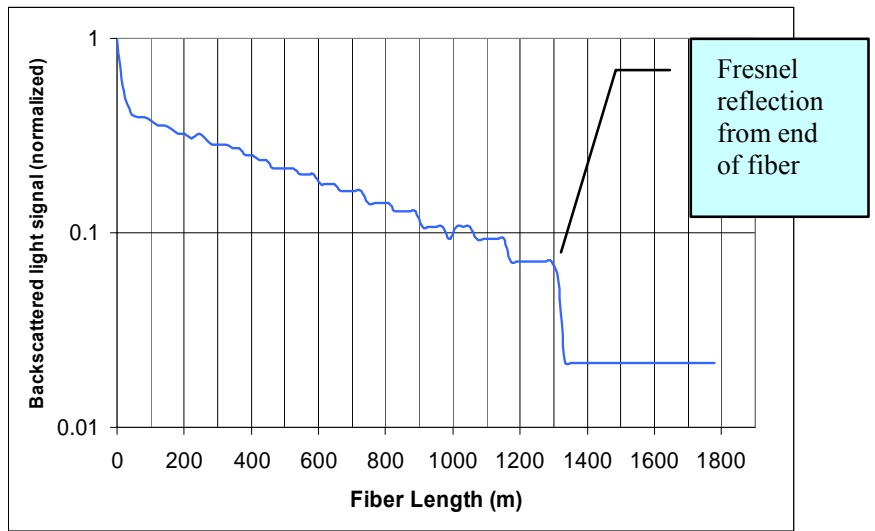

Fig. 3. Logarithmical division of the backscattered signal versus fiber length

The typical result obtained for the measurement of the optical fiber attenuation using a continuous wave with a beam coupler is shown in Figure 4. The total loss for the whole system is $10.08 \mathrm{~dB}$. The loss at the optical coupler is $3.05 \mathrm{~dB}$ and the loss at the fusion splice is $0.25 \mathrm{~dB}$. By subtracting the external loss from the system, the total loss through the fiber for two way flows was obtained as 6.78 $\mathrm{dB}$. The attenuation coefficient, $\alpha_{d B}$ could be determined as:

$$
\alpha_{d B}=\frac{\text { Total Loss }(\mathrm{dB})}{\text { Distance }(\mathrm{km})}
$$

From the calculation the value of the attenuation coefficient, $\alpha_{d B}$ is $(2.58 \pm 0.02) \mathrm{dB} \cdot \mathrm{km}^{-1}$.

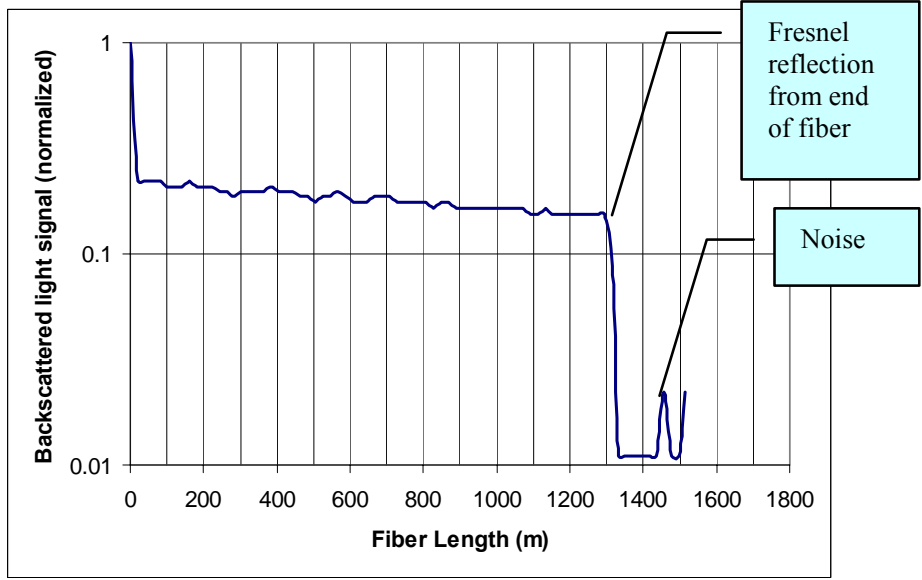

Fig. 4. Logarithmical division of the backscattered signal versus fiber length

The measured value of attenuation coefficient by using MEOS OTDR with a beam splitter yields a higher value by $0.07 \mathrm{~dB} \cdot \mathrm{km}^{-1}$ or $2.71 \%$ compared to beam coupler. Whereas the average value for the MEOS OTDR is 2.62 $\mathrm{dB} \cdot \mathrm{km}^{-1}$. In comparison with Anritsu OTDR the measured difference could be achieved upto $14.91 \%$. Thus the attenuation coefficient measurement by using Anritsu OTDR is lower in comparison to MEOS OTDR.

The measurement of fiber length by using pulsed laser from MEOS OTDR which utilized a beam splitter and an optical beam coupler yields a difference of $0.77 \%$. The average measurement of MEOS OTDR optical fiber length yields an excess of $4 \mathrm{~m}$ or $0.31 \%$ when compared with Anritsu OTDR.

The results obtained for the measurement of coupling efficiency and backscattered signal due to the presence of BK7 glass window has been tabulated in Table 1. The graph of measured output voltage versus rotation angle is plotted as Figure 5.

Table 1: Coupling voltage and backscattered voltage at Port 2, 3

\begin{tabular}{|c|c|c|c|}
\hline $\begin{array}{c}\text { Angle } \\
\left(\boldsymbol{\theta}^{\mathbf{0}}\right)\end{array}$ & $\begin{array}{c}\text { Port 2 } \\
(\mathbf{V} \pm \mathbf{2}) \mathbf{~ m V}\end{array}$ & $\begin{array}{c}\text { Port 3 } \\
(\mathbf{V} \pm \mathbf{2}) \mathbf{~ m V}\end{array}$ & $\begin{array}{c}\text { Port 4 } \\
(\mathbf{V} \pm \mathbf{2}) \mathbf{~ m V}\end{array}$ \\
\hline 0 & 13.4 & 15.2 & 4.2 \\
\hline 5 & 14.4 & 16.2 & 7.6 \\
\hline 10 & 15.6 & 17.0 & 10.4 \\
\hline 15 & 16.0 & 17.8 & 14.0 \\
\hline 20 & 16.8 & 18.2 & 16.0 \\
\hline 25 & 17.2 & 18.6 & 16.6 \\
\hline 30 & 17.2 & 18.4 & 16.8 \\
\hline 35 & 17.4 & 18.6 & 17.8 \\
\hline 40 & 17.0 & 18.6 & 16.8 \\
\hline 45 & 16.2 & 18.2 & 12.6 \\
\hline 50 & 13.6 & 15.4 & 5.4 \\
\hline 55 & 11.0 & 16.4 & 1.2 \\
\hline
\end{tabular}




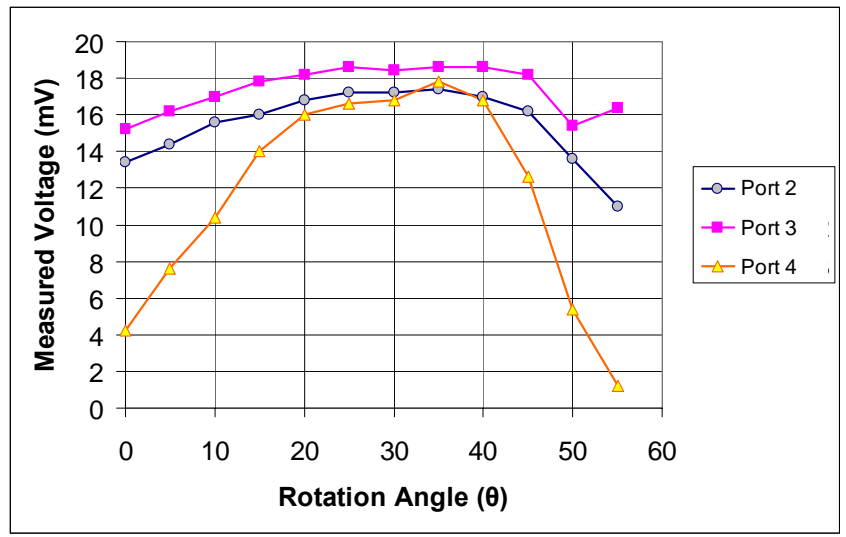

Fig. 5. Measured Voltage versus Rotation Angle

Figure 5 shows that the optimum coupling voltage at the Port 2 and Port 3 occurs around $35^{\circ}$ rotation of the BK7 glass window in the anticlockwise direction. This interesting phenomenon requires further investigation.

\section{CONCLUSION}

The OTDR system was successfully modified by using a fiber coupler. The length and attenuation measurement of fiber optic were measured and compared with a conventional Anritsu OTDR. It is also observed that the presence of a BK7 glass window in front of the coupling optics increases the coupling efficiency.
The multimode optical fiber length is obtained as $(1313.99 \pm 21.10) \mathrm{m}$ with the calculated attenuation coefficient of $(2.65 \pm 0.03) \mathrm{dB} \cdot \mathrm{km}^{-1}$ which measured using a polarized beam splitter. The measurement using a beam coupler yields a fiber length of $(1303.89 \pm 10.99) \mathrm{m}$ with the calculated fiber attenuation of $(2.58 \pm 0.02) \mathrm{dB} \cdot \mathrm{km}^{-1}$. The deviation of fiber length and attenuation between the two methods are $0.77 \%$ and $2.71 \%$. The fiber attenuation coefficient results comply with the specifications stated in the manufacturer's brochure and are in the acceptable region for modern graded index multimode fiber as stated by Harry J.R Dutton $[8,9]$. The beam coupler provides better accuracy with an acceptable tolerance compared to a polarized beam splitter. The OTDR device can be designed in compact, rugged and low cost by utilizing the fiber coupler.

This measurement, however varies with the actual measurement using Anritsu OTDR by an average of 14.69 $\%$ for the attenuation coefficient measurement and also by an average of $0.42 \%$ for fiber length. This phenomenon shows that attenuation coefficient is highly influenced by the light source's wavelength [10].

An adjustable BK7 glass plate window provides a signal optimization in the system when rotated $35^{\circ}$ anticlockwise. This window provides a cheap solution for coupling the source into the fiber with high efficiency and by amplifying the input power.

\section{REFERENCES}

[1] G. Keiser. Optical Fiber Communications. $3^{\text {rd }}$ edition. Singapore: McGraw-Hill Higher Education. 2000.

[2] M. Tateda and T.Horiguchi. Advances in Optical Time-Domain Reflectometry. Journal of Lightwave Technology. 1989.8 (7) : $1217-1224$.

[3] B. J. Stinson. Design and Test Of An Event Detector And Locator For The Reflectoactive Seals System. M.Sc Thesis. The University of Tennessee, Knoxwill; 2006.

[4] J.C. Palais. Fiber Optic Communications. $4^{\text {th }}$ edition. New Jersey, U.S.A: Prentice-Hall. 1998.

[5] S.O Kasap. Optoelectronics and Photonics. Canada: Prentice-Hall, 2001.

[6] D. L. Phillen, Ian A. White, Jane F. Kuhl and Stephen C. Mettler. Single-Mode Fiber OTDR: Experiment and Theory. IEEE Journal Of Quantum Electronics. 1982. 10 (18): 1499-1508.

[7] J. Crisp. Introduction to Fiber Optics. Britain : Newnes. 1996.

[8] J- Fiber GmbH. Wide Grade Multimode Fiber 50/125. Jena, Germany : Trade brochure. 2005.

[9] H. J. R. Dutton. Understanding Optical Communications. U.S.A : IBM-International Technical Support. 1998.

[10] K.I. Aoyama, K. Nakagawa, and T. Itoh. Optical Time Domain Reflectometry in a Single-Mode Fiber. IEEE Journal of Quantum Electronics. 1981. 6 (17): $862-868$ 